\title{
Notfall-Analgesie
}

\section{Bei Nierenkolik NSAR statt Opioid geben?}

Die gängige Praxis, bei Nierenkolik ein Opioid i.v. zu geben, ist offenbar nicht optimal. Eine wirksamere Schmerzlinderung wurde in einer randomisierten, kontrollierten Studie mit der i.m.-Applikation eines nichtsteroidalen Antirheumatikums (NSAR) erreicht.

Die Präferenz für intravenöse Opioide zum Akut-Management von Nierenkoliken beruht auf der Annahme, dass damit eine schnellere Resorption und eine effektivere Schmerzreduktion zu erzielen ist als mit einer intramuskulären Analgesie. Eine doppelblinde, randomisierte und kontrollierte Studie stellt diese Auffassung jedoch infrage. Für einen Großteil der Patienten, die mit Nierenkolik eine Notaufnahme aufsuchen, könnte ein NSAR i.m. demnach das Analgetikum der Wahl sein. Wie die Studienautoren aus Katar und Australien berichten, war intramuskuläres Diclofenac sicher und sorgte, im Vergleich $\mathrm{zu}$ Morphin i.v. und Paracetamol i.v., für die schnellste, wirksamste und anhaltendste Schmerzlinderung.

An der Studie waren 1.644 Notaufnahme-Patienten mit V. a. Nierenkolik beteiligt. Die Schmerzen hatten sie auf einer numerischen Rating-Skala (NRS) von 1-10 im Median mit 8 Punkten bewertet. 547 Teilnehmer erhielten das NSAR Diclofenac (75 mg/3 ml i.m.), 548 Paracetamol (1 g/100 ml i.v.) und 549 Morphin (0,1 mg/kg KG i.v.). Um die Studientherapie verblinden $\mathrm{zu}$ können, bekamen alle Patienten drei Injektionen, eine mit Wirkstoff und zwei mit Placebo.

\section{Schnellere Schmerzreduktion}

Eine Schmerzreduktion von mindestens $50 \%$ nach 30 Minuten, das primäre Studienziel, wurde mit Diclofenac von 68\%, mit Paracetamol von 66\% und mit Morphin von $61 \%$ der Patienten erreicht. Der Unterschied von Diclofenac, aber nicht der von Paracetamol zu Morphin war signifikant.

Wurde die Auswertung auf diejenigen Patienten beschränkt, bei denen per CT oder Ultraschall Harnleitersteine nachgewiesen werden konnten ( $\mathrm{n}=1.316)$, betrugen die Anteile der Patienten mit mindestens halbierter Schmerzintensität $69 \%, 68 \%$ und $60 \%$. Unter dieser Voraussetzung waren sowohl Diclofenac i.m. als auch Paracetamol i.v. der Morphingabe überlegen.

\section{Länger anhaltende Wirkung und seltenere Nebenwirkungen}

Die NSAR-Gruppe benötigte 30 Minuten nach der ersten Analgesie seltener eine Rescue-Analgesie mit demselben Wirkstoff als die beiden anderen Gruppen (12\% vs. $20 \%$ bzw. $23 \%$ ). Auch nach 60 Minuten war der Anteil der Patienten mit

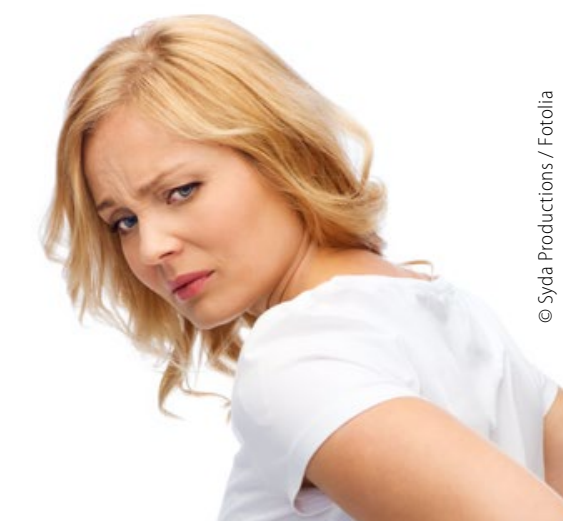

Bei Schmerzen durch Nierenkolik ist eine schnelle und effektive Analgesie erforderlich. persistierenden Schmerzen (NRS > 2) unter Diclofenac am geringsten (24\% vs. $30 \%$ bzw. $38 \%$ ).

Unerwünschte Ereignisse wurden unter Diclofenac und Paracetamol seltener registriert als unter dem Opioid (1\% vs. $1 \%$ vs. $3 \%$ ). Bei einer Nachuntersuchung zwei Wochen später wurde in der NSARGruppe zwar häufiger ein erhöhtes Serumkreatininwert festgestellt als unter Morphin. Bis zur vierten Woche hatten sich aber alle Werte normalisiert. Kein Patient musste zur Dialyse.

\section{Patienten mit Niereninsuffizienz ausgeschlossen}

Nach Ansicht der Studienautoren um Sameer Pathan „widersprechen die Ergebnisse der klinischen Glaubenslehre, wonach eine intravenöse Analgesie, zumal mit Narkotika, schneller und effektiver ist“. Die Resultate hätten daher „tiefgreifende Konsequenzen“ für die Erstversorgung von Nierenkolikpatienten: „NSAR i.m. sollten als Erstlinientherapie betrachtet werden." Die Sorge, durch eine einmalige NSAR-Injektion Komplikationen wie Muskelnekrosen, MagenDarm-Blutungen oder Dialysepflicht zu verursachen, sehen die Ärzte aufgrund ihrer Untersuchung als weitgehend unbegründet an.

In diesem Zusammenhang ist allerdings unbedingt $\mathrm{zu}$ beachten, dass $\mathrm{Pa}$ -

tienten mit Niereninsuffizienz von der Studie ausgeschlossen waren, ebenso Patienten mit Leberschäden, Asthma oder Schwangerschaft. Sie werden daher von Pathan und Kollegen ausdrücklich von der Empfehlung zur NSAR-Gabe ausgenommen.

(Beate Schumacher)

Pathan SA et al. Delivering safe and effective analgesia for management of renal colic in the emergency department: a double-blind, multigroup, randomised controlled trial. Lancet 2016, online 15. März; doi: 10.1016/S0140-6736(16)00652-8 